\title{
How to improve the interaction design of NFC payment terminals?
}

\author{
Poornigha Santhana Kumar ${ }^{1}$ Michael Bechinie ${ }^{1}$ and Manfred Tscheligi ${ }^{2}$ \\ ${ }^{1}$ USECON, 1110 Vienna, Austria \\ kumar@usecon.com, bechiniedusecon.com \\ ${ }^{2}$ University of Salzburg, 5020 Salzburg, Austria \\ manfred.tscheligi@sbg.ac.at
}

\begin{abstract}
Near field communication (NFC) payments also popularly known as contactless payments are increasingly used in retails shops like supermarkets and cafes nowadays. User studies on NFC payments show that NFC payments fail to provide users with the appropriate user experience. In this paper, we aimed to redesign the existing NFC payment experience design by altering 5 factors namely the audio feedback, the visual feedback, the haptic feedback, the screen design of the payment terminal and the NFC component. The results show that altering the aforementioned factors increase the usability of NFC payments and provides users with privacy and security-enhanced experience. We also framed 5 guidelines based on our evaluation results which will aid designers while designing or redesigning any system. We also believe that our research methods and guidelines contribute to the researches in the HCI community.
\end{abstract}

Keywords: NFC payments, Usability, User experience, Lived Experience, Design guidelines

\section{Introduction}

User experience plays an important role in the systems/services/products we use in our everyday life. Each system we interact with leaves us with experience [1] [2]. This experience plays a vital role in the success of the system. For a system to be successful it should be designed considering 3 factors. The usability [4] of the system, the user experience [4] gained by the users from the system and the lived experience [1]. Figure 1 represents the relationship between usability, user experience and lived experience.

The different payment terminals used widely can be categorized into 4 main overlapping categories based on the feedback and hardware design. Namely,

Visual feedback on screen with audio feedback - The visual feedback on the state of the transaction and the visual feedback on the success of the transaction is displayed on the screen. The visual feedback is accompanied by sound feedback

Visual feedback on hardware with audio feedback - The visual feedback on the state of the transaction and the visual feedback on the success of the transaction is displayed via lights in on the payment terminal hardware. The visual feedback is accompanied by sound feedback 
NFC scan pad on the screen - Payment terminals which lets users scan their NFC component on the side of the terminal. This terminal also delivers users with visual and audio feedback

NFC scan pad on the side of the terminal - Payment terminals which lets users scan their NFC component on the screen of the terminal. This terminal also delivers users with visual and audio feedback

All the above-mentioned terminal were initially designed for credit/debit card payments and were adapted to NFC payments. These terminals do not completely support NFC payments on scales of usability and user experience. NFC cards are in use for payments in the form of NFC cards since 2007 in the UK. This payment technology enables users to pay by simply tapping their NFC enabled component against any active payment terminal [5]. NFC technology is known for its fast interaction compared to existing interaction methods [6].

Researches have been carried out on NFC's security [7], acceptance [8] [9] and applications [5]. Only a few studies have been conducted on scales of usability on NFC. Research [10] [11] on NFC payments in supermarkets shows that there is a lack of usability and appropriate user experience in NFC payment in supermarkets. In this paper, we demonstrate how we addressed various factors of the payment terminal to support NFC payments and to improve its usability, user experience and lived experience of NFC payments.

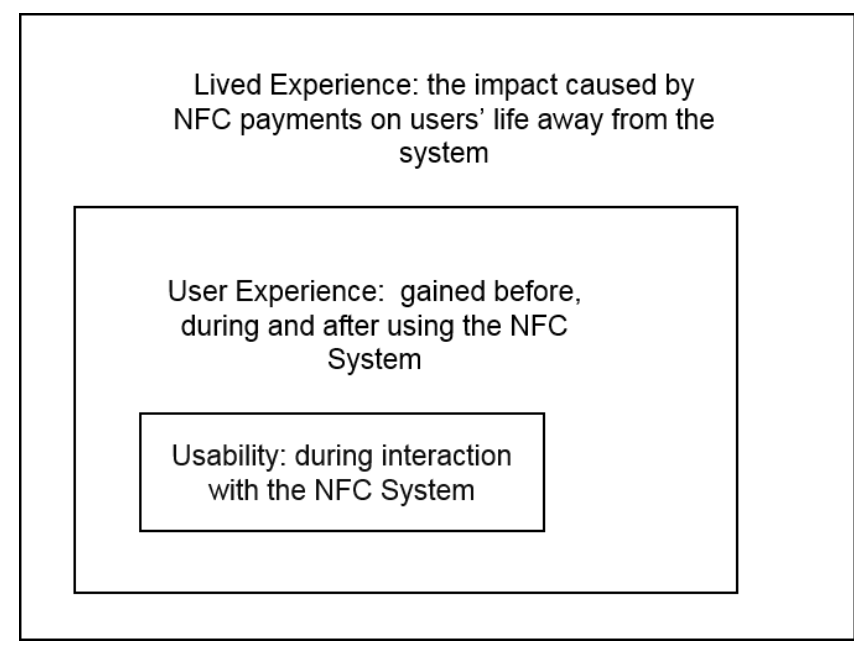

Fig. 1. The relationship between usability, user experience and lived experience of NFC payments

\section{Method}

We are following the well-established UCD [3] process throughout our project. As a first step, we conducted a user study to understand the existing practices and difficulties 
of NFC payments [10][11]. During the user study, we also observed the design of payment terminals used in various supermarkets and cafes in Austria, Germany, Switzerland and the UK. We chose to work with supermarkets and cafes as a wide range of customers uses it on a daily basis. Our user study results portrayed that users feel less secured while paying with NFC compared to other payment methods. So to improve the usability and privacy \& security related experience of the user, we redesigned the existing payment terminal.

\subsection{Prototyping}

We redesigned the payment terminal based on the user study data and the heuristics principles [12]. Five factors were considered for redesigning the prototype. We considered the possible feedbacks namely: visual feedback, audio feedback and haptic feedback. Additionally, the screen design of the payment terminal and the NFC component used to pay was also considered. To evaluate the effect of each factor on the usability and the user experience gained by the users, we designed prototypes corresponding to each factor. All the payment terminal prototypes were sized $(19 \mathrm{x} 9 \mathrm{~cm})$ to the real world payment terminals.

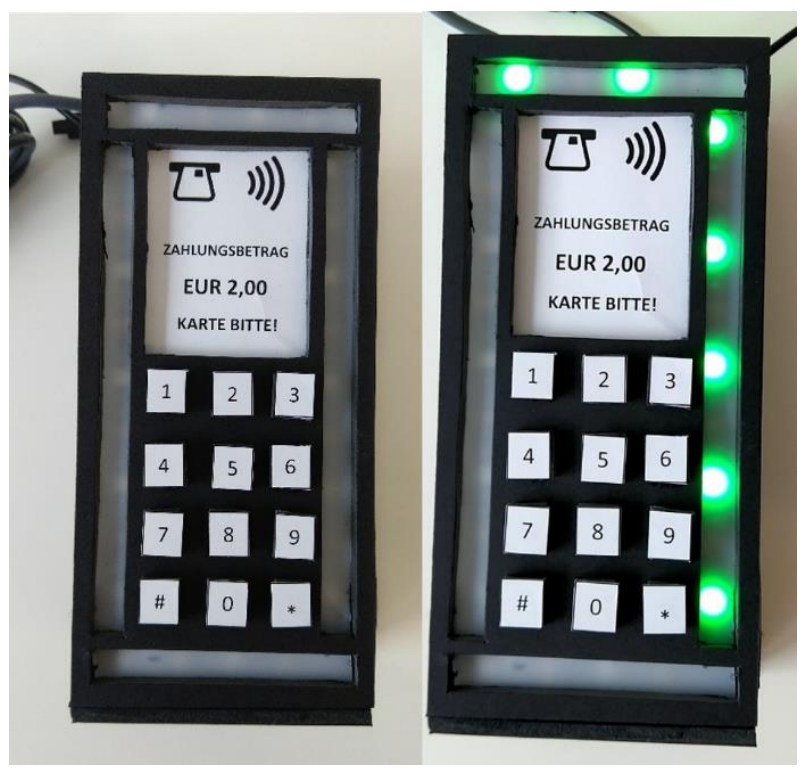

Fig. 2. a) Visual feedback prototype b) visual feedback prototype with LEDs lighting in a clockwise direction to indicate the state of the transaction

\section{Audio feedback.}

Most existing payment terminals use sound feedback to notify the user. This sound feedback is not consistent in the pitch, length of the sound and the pattern across the supermarkets. In most supermarkets, the payment terminals provide audio feedback 
throughout the transaction as a long beep. In our prototypes, we used two different types of audio feedback

Audio feedback only at the end of the transaction - short beep sound at the end of the transaction

Audio feedback at each stage of the transaction - beep sound corresponding to the loading bar and at the end of the transaction

\section{Visual feedback.}

One of the main disadvantages in adapting the credit/debit card terminals for NFC payment is the visual feedback provided on the screen is actively hidden by the users when scanning the NFC component against the payment terminal while paying. This makes the users lack feedback regarding the end of the transaction which leads to the users holding their NFC components against the payment terminal longer than it is required. NFC payments are known for being quicker than other payments. Since the lack of visual feedback obstructs this advantage, existing visual feedback needed to be improved. In our prototype (Figure 2.a), we placed the visual feedback on the hardware of the payment terminal. Payment terminals are used in environments which tend to be noisy like in cafes and supermarkets. As we cannot rely on audio feedbacks in such environments we also increased the size of the visual feedback. Green LED lights were placed around the payment terminal which acts as a loading bar during the transaction. The LED lights will be lit in a clockwise direction to show the state of the transaction to the users (Figure 2.b).

Taking lived experience into account, the LED lights will act only as the feedback on the state of the transaction which in turn shows how long the users need to hold their card against the terminal. The success or failure of the transaction will be displayed only on the screen of the terminal. If the transaction fails the LED lights will not turn red. This is to prevent the negative lived experience gained by the users while using NFC payments in public places. Having a transaction declined in a public place might lead the user into never trying NFC payments again. 


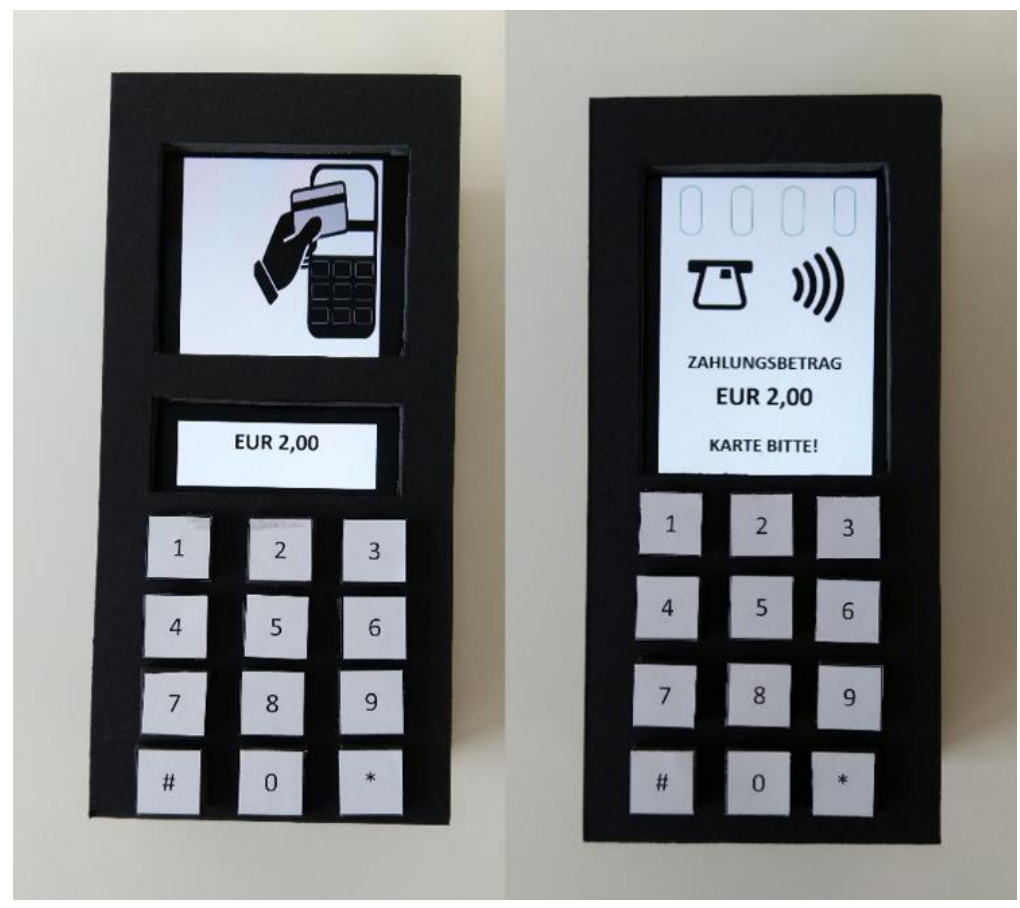

Fig. 3. a) Screen design prototype with a modified screen design. b) Base prototype - replication of the payment terminals used in supermarkets and cafes.

\section{Haptic feedback.}

We aimed to redesign the payment terminal such that it will support all the customers. We included haptic feedback to support any differently-abled peopled using the payment terminal. Differently abled customers may not be able to see the visual feedback or hear the audio feedback. So adding haptic feedback will help them feel the feedback and understand the state of the transaction. A vibrate feedback was added at the end of the transaction as feedback.

\section{Screen design.}

As explained in visual feedback, the feedback provided on the screen is hidden by the NFC component. To avoid this we redesigned the screen design of the payment terminal (Figure 3.a). We split the screen into 2 parts. The top section is to scan the NFC component and the bottom section is to see the feedback provided by the payment terminal. The visual feedback is used to portray the state of the transaction and the bottom section is used to provide feedback on the end of the transaction. The bottom section of the screen shows the users if the transaction is successful or declined in a discreet manner without causing any social embarrassment thereby leaving them with positive lived experience. 


\section{NFC Component.}

The type of NFC component used by the users influences the user experience gained by the users and these components also differ on scales of usability. We considered 3 NFC components in total (Figure 4). First, the commonly used NFC component, the NFC debit cards and credit cards. The second NFC component considered was NFC mobile app. With apple pay and android pay mobile NFC is also spreading across many countries. We also considered the lesser-known NFC sticker as the third NFC component. The prototypes were made to match the real world dimension. The NFC card, NFC mobile app and NFC sticker measured $8.5 \times 5.5 \mathrm{~cm}, 14 \times 7 \mathrm{~cm}$ and $4 \times 3 \mathrm{~cm}$ respectively.

The prototypes were developed corresponding to each factor and other aspects of the payment terminal were not changed. In visual feedback prototype, a prototype of the payment terminals used in supermarkets was created and only the visual feedback in the payment terminal was replaced by our visual feedback. Other features like the audio feedback and screen designs were not modified. Similarly, for screen design prototype, the screen design of the payment terminal was replaced by our screen design. The visual feedback and audio feedback were not modified. Using the same procedure haptic and audio feedback prototypes were also developed. In addition to these prototypes, we also replaced the payment terminals used in supermarkets as a prototype to act as our base terminal (figure 3.b).

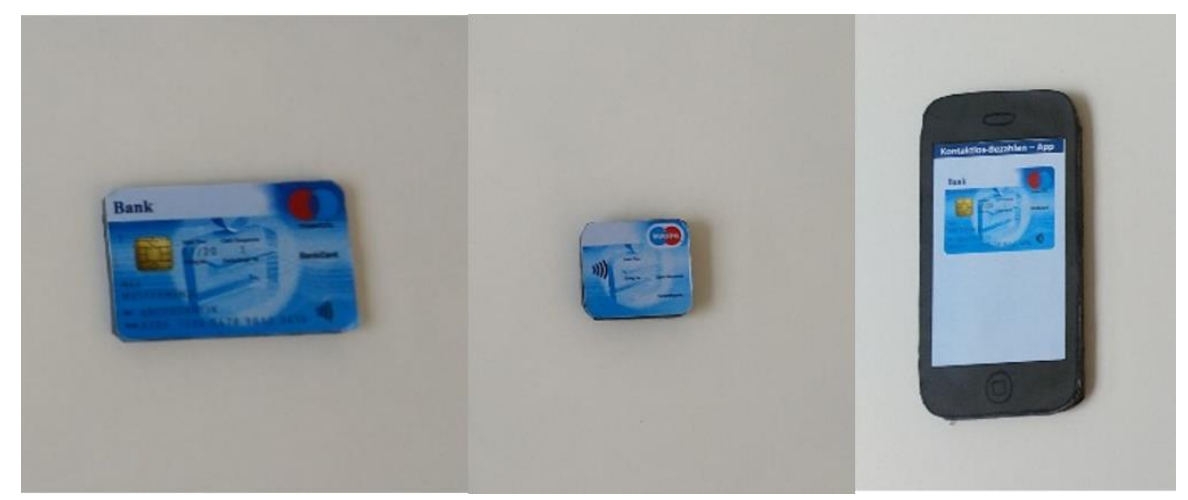

Fig. 4. NFC components a) NFC card b) NFC sticker c) NFC mobile App

\section{Evaluation}

The usability and user experienced gained from each prototype was evaluated during the evaluation phase. To measure the usability of the prototypes UMUX questionnaire [19] was used. The evaluation took place in Austria, a German-speaking country. To support our participants the standard UMUX questionnaire was translated to German by professional translators. The translation was back-translated to English by professional translators and was checked for equivalence to the original questionnaire. 
To evaluate the privacy and security related user experience gained, a Likert scale was used. Any user paying with a payment terminal would prefer to feel secure. We aimed to improve the user experience gained by the users by delivering the users with a secured experience while paying with NFC. The users were questioned "How secured did they feel while paying with prototype a/b" and they were asked to rate their experience on a 1-5 (1 - low, 5 - high) Likert scale. We asked a simple question instead of using any standard privacy and security scales because we aimed to measure only the felt privacy and security user experience of the user.

Participants were recruited via USECON database (www.askus.at) for evaluation. Participants who have experience with NFC payments were recruited for evaluation and an incentive of $30 €$ was given to each participant. During the evaluation, the participants were asked to pay with an NFC debit card (prototype - Figure 4.a) with the base terminal and any one of the redesigned prototypes say the visual feedback prototype. Then they were asked to fill out the UMUX and Likert scale questionnaire for each prototype. After filling out the questionnaires the participants were asked an open question on their liking/disliking of the prototypes. Then the participants were again asked to pay with an NFC debit card (prototype - Figure 4.a) with the base terminal and any one of the redesigned prototypes say the audio feedback prototype followed by the questionnaires. This procedure was repeated for all the redesigned prototypes. The participants were asked to pay with only NFC card as it is the commonly used NFC payment component.

To evaluate the NFC component, the participants were asked pay with NFC card, NFC mobile app and NFC sticker on the base terminal. To provide participants with close to real-time feeling during evaluation they were asked to open the NFC application in the prototype prior to paying while paying with NFC mobile app. For NFC stickers, they were asked to choose anyone everyday object prototype (Figure 5) and were asked to attach the NFC sticker to the object before paying with NFC sticker. The evaluation was then followed by questionnaires and open question.

During the evaluation, the order in which the prototypes were tested was changed for each participant to avoid order bias.

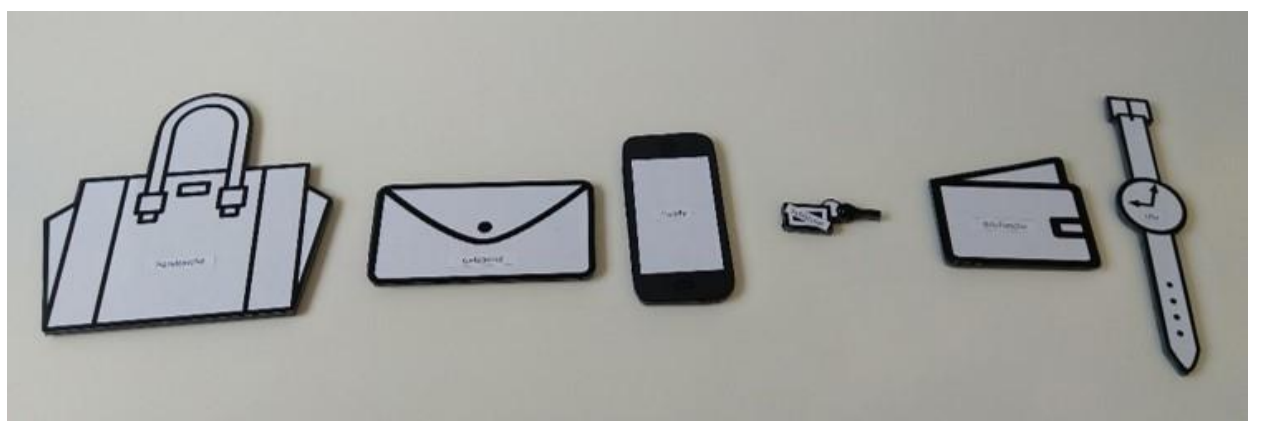

Fig. 5. Prototypes of everyday objects considered and used while evaluating NFC sticker 


\section{$4 \quad$ Results}

Out of 33 participants, 14 were female and 19 were male with the mean age of 31 . All the participants had prior experience with NFC payments. The UMUX scores represent the usability of the prototype and the Likert scale values represent the user experience gained from the prototype.

\subsection{Usability results}

UMUX score for all the redesigned prototypes and base prototype was calculated. Figure 6 shows a comparison of mean UMUX scores of the prototypes and corresponding base terminals. The UMUX scores were compared by the Wilcoxon Signed-Rank test. The results show that there is a significant difference between the base prototype and redesigned prototypes. The visual feedback prototype and screen design prototype were significantly better than the base prototype with $\mathrm{p}<0.05$, z-value -2.941 and -2.5495 respectively.

The UMUX scores of the audio feedback show that the existing audio feedback (long beep throughout the transaction) is better than the redesigned audio feedbacks. The base terminal was significantly better than the audio feedback only at the end of the transaction and audio feedback at each stage of the transaction prototypes with $\mathrm{p}<0.05, \mathrm{z}-$ value -2.5205 and -3.0594 respectively.

NFC components' UMUX score portrays that NFC card is usable compared to NFC mobile app and NFC sticker. The NFC card is significantly better than NFC mobile app and NFC sticker with $\mathrm{p}<0.05$, z-value -3.1798 and -2.6656.respectively.

The haptic feedback prototype's UMUX scores (92.646) were similar to the base terminal's UMUX scores (93.137) with no significant difference. When questioned about their liking, 29 out of 33 participants felt the haptic feedback to be neutral. One participant mentioned that he/she thought it was his mobile phone as he always keeps his mobile on vibrate mode. Another participant said he/she did not like the vibrate feedback as it sounded like an error. 


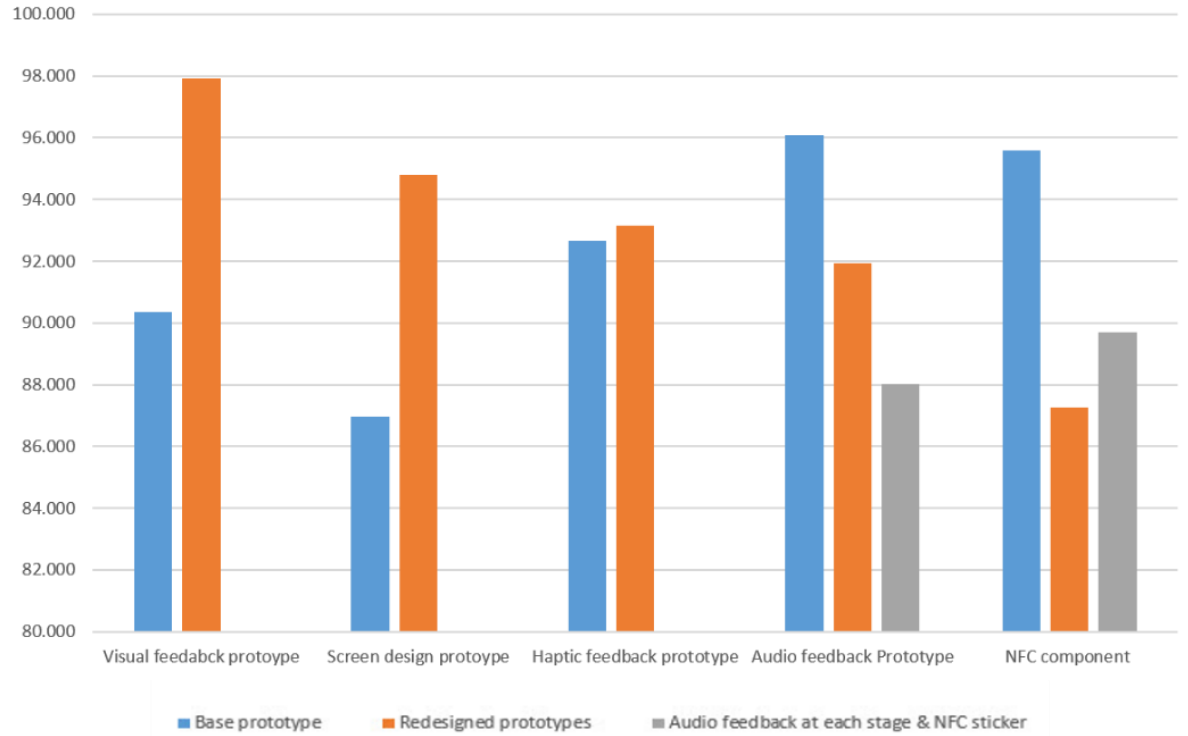

Fig. 6. Representing the difference between the mean of UMUX scores of base prototypes and the redesigned prototypes of each factor. The blue bars represent base prototype, orange bars represent the redesigned prototypes. The grey bars represent the prototype with audio feedback at each stage of the transaction and NFC sticker.

\subsection{Results for felt security}

The mean of Likert scale values was calculated for all the prototypes. These values were compared to know users' perceived security related experience from the prototypes. The user experience results were directly proportional to the usability results showing that usable prototypes deliver users with the appropriate user experience.

Participants felt secured while paying with the redesigned visual feedback and screen design prototype compared to the base terminal. Similar to UMUX score the base prototype was considered to be secured compared to other audio feedback prototypes. For haptic feedback, both the base terminal and the haptic feedback terminal had the same mean Likert score demonstrating that there is no difference in the experience gained. NFC cards provide users with secured user experience compared to NFC mobile app and NFC sticker. Figure 7 shows the mean Likert scale values of the prototypes and corresponding base terminals. 


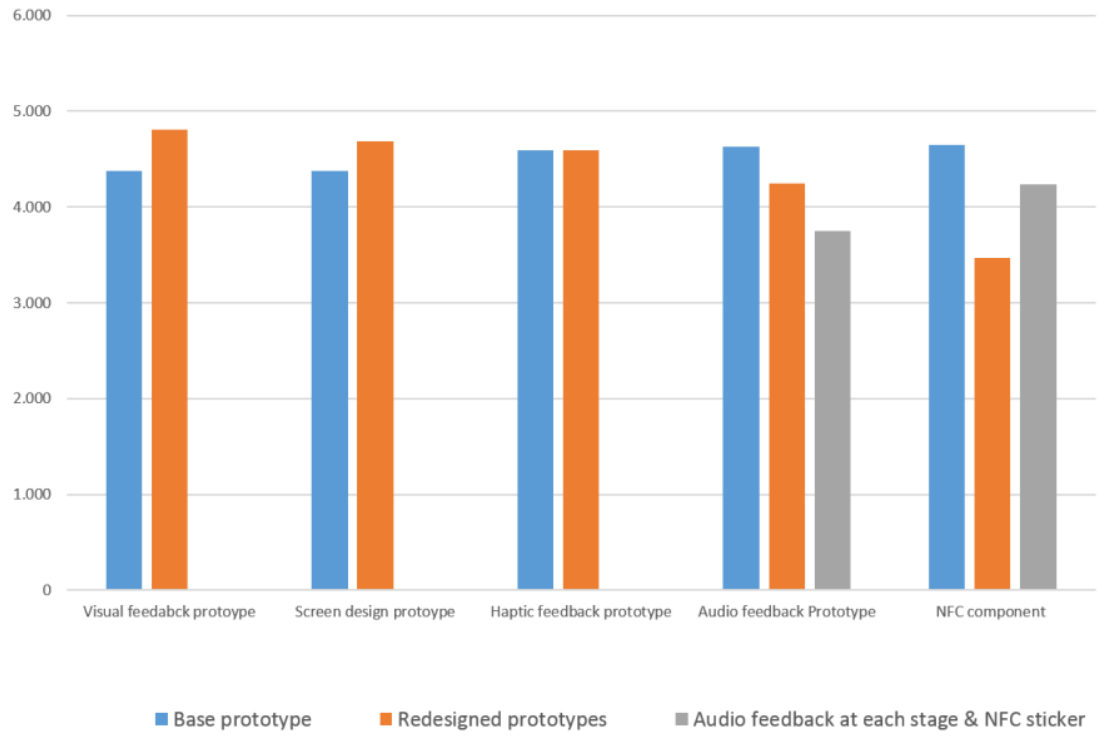

Fig. 7. Representing the difference between the mean of Likert scales values of base prototypes and the redesigned prototypes of each factor. The blue bars represent base prototype, orange bars represent the redesigned prototypes. The grey bars represent the prototype with audio feedback at each stage of the transaction and NFC sticker.

\section{Iteration}

We designed a payment terminal comprising of all the factors which were usable and delivered users with security-enhanced experience. The redesigned prototype had the split screen design and visual feedback on the hardware of the payment terminal. Even though haptic feedback did not have an effect on usability and user experience, we added the haptic feedback to the redesigned payment terminal to support differentlyabled customers. The audio feedback used in supermarkets was used without modification. Figure 8 shows the redesigned payment terminal. This payment terminal was then reevaluated against the base terminal on the scales of user experience provided.

The reevaluation was conducted in Austria and the participants were recruited directly from cafes and university campuses. In total, we had 113 participants out of which $71(63 \%)$ were male and $42(37 \%)$ were female with a mean age $=35$. All the participants had prior experience with NFC payments. The participants were asked to pay with NFC card (prototype - Figure 4.a) on the redesigned payment terminal and the base payment terminal. They were then asked to rate their felt of security on a 1-5 Likert scale for each terminal. Finally, they were asked to share any comment regarding the payment terminals. Again the order in which the prototypes were tested was changed to avoid order biases. 
The mean of the Likert scales values was compared using the Wilcoxon SignedRank test. The results demonstrated that the redesigned payment terminal provides significantly better user experience than the base payment terminal with $\mathrm{p}<0.01, \mathrm{z}-$ value -8.2164. Below are some answers from participants showing that the features in the redesigned payment terminal help them feel secured while paying with NFC.

"It vibrates, I need not even look into the screen I will know it"

"The lights are visible compared to the other one (base prototype)"

"I can see the amount while paying that make me feel secured"

"The vibration feature will help in supermarkets because it (the supermarkets) is usually noisy"

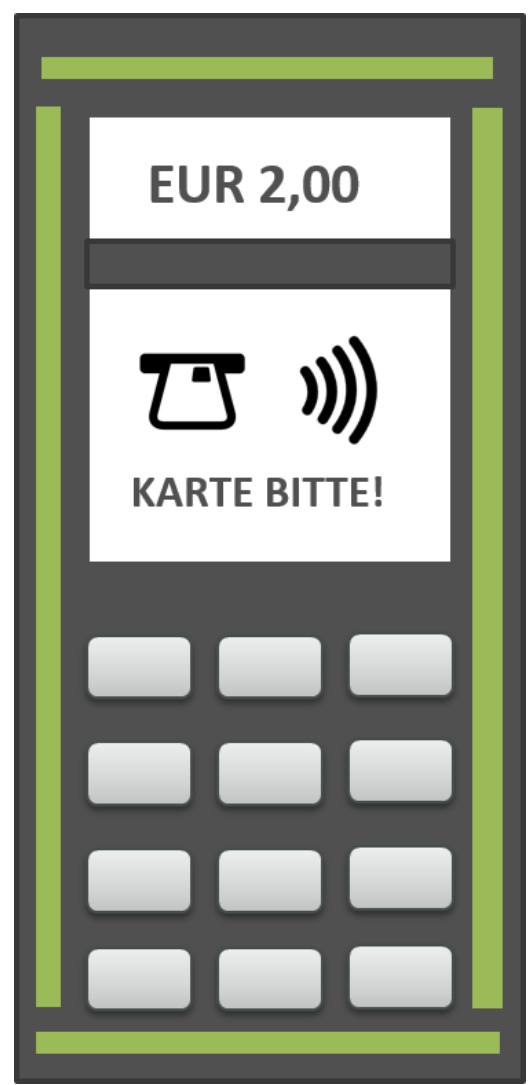

Fig. 6. Final payment terminal design

\section{Discussion}

The evaluation and reevaluation results clearly show that the redesigned payment terminals had a higher degree of usability and provide users with a secured experience. To generalize our findings and to contribute to the designing or redesigning process of any system/service/product, we framed 5 guidelines based on our evaluation results. 


\section{Redesigning is crucial}

Adapting an existing system for a different purpose may solve the need but it will not be successful as it is not developed for that particular purpose. The system should be redesigned based on the new needs to fully support the aroused need. Skipping the user analysis phase leads to usage problems. Adding a component to a system changes the context of use, so user analysis should be performed to ensure usability and appropriate user experience in the later design. In our case, the payment terminals designed for debit/credit card payments were adapted to support NFC payments in recent years. The adaptation allows users to pay with NFC components but this adaptation is not completely successful as it has usability and user experience flaws. A system should be redesigned for timely needs for successful functioning.

\section{Visual feedback is key}

Visual feedback plays an important role in any system. This is already stressed in the literature by many guideline sets [12][13]. During the evaluation of our prototypes, we found that the visual feedback prototype was perceived to be the most usable and secured prototype. We should try to provide visual feedback on everything that happens in the system as human beings rely on visual feedback more than other feedback forms. Especially when designing or redesigning payment-related systems like payment terminals, online checkout pages and ticket machines, information like amount to be paid, the status of the transaction and the result of the transaction should be visually communicated to the users.

\section{Black \& white vs. colour interfaces}

Colour has been studied in various directions in the literature. Psychological studies [14] and marketing studies [15] shows that certain colours trigger some emotions. [16] shows that colour also has an effect on human performance. In HCI, the law of simplicity states [17], when considering emotions we need to add components to achieve it. In our case, the considered emotion was triggered by coloured interfaces. One of our payment terminal interface was black \& white. Users perceived the black \& white interfaces to be outdated thereby considering it less secured. Adding some colour to the interfaces gave users more secured and updated feel. While designing any system, we should consider adding colour to the interfaces if applicable.

\section{Failure of the system does not imply the failure of feedback}

A system might not be usable and deliver users with appropriate experience. This does not imply that the feedbacks used by the system is also not usable. In our case, the payment terminals used in the supermarkets were not usable for NFC payments and did not deliver them with the secured user experience. But the audio feedback used by these payment terminals did provide users with secured experience compared to other alternate audio feedbacks. Users might be used to certain feedbacks of the system which cannot be replaced. So while redesigning any system or designing any new system, each feedback from an old system or similar system should be considered. 


\section{Neutral feedbacks for efficient designing}

All feedback from a system need not be useful/informative for all users. Some types of feedback can be added in a way such that it is subtle for some users and dominant for other users. In our case, the haptic feedback did not add any value to users and was considered to be neutral. Haptic feedback will aid differently-abled people and will act as important feedback. Instead of designing a new system to support differently-abled users, we should try and incorporate neutral features into standard systems. This is not only economical but also helps to avoid the effect of exclusion and isolation [18] on differently abled users.

\section{$7 \quad$ Limitations}

The user studies were conducted in supermarkets in real-time whereas the evaluations were conducted in a lab setting. During the evaluation, we tried to recreate the real-time environment by giving them a supermarket scenario before testing the prototypes. Still the results acquired from lab setting might slightly vary when tested in real-time.

\section{$8 \quad$ Next steps and conclusion}

As a next step, we aim to achieve a universal design for the payment terminal. The redesigned payment terminal will be evaluated with differently abled participants and will be iterated according to needs. We will also update the guidelines list based on the evaluation results with the differently-abled people.

To conclude, the payment terminals used in supermarkets and cafés do not support NFC payments on scales of usability due to lack of lucid feedback. It also fails to provide users with an appropriate user experience which makes the users consider NFC payments to be less secured compared to other payments methods. We redesigned 3 factors namely visual feedback, haptic feedback and screen design of the payment terminal to support NFC payments. We also evaluated 2 other factors of NFC payments, the NFC component (NFC card, NFC mobile app and NFC sticker) and the audio feedback. Based on the evaluation results, we framed 5 guidelines for designing or redesigning a system to aid designers and other fellow researchers.

\section{Acknowledgement}

The project leading to these results has received funding from the European Union's Horizon 2020 research and innovation program under the Marie Sklodowska-Curie grant agreement No 675730 . 


\section{Reference}

1. McCarthy, John, and Peter Wright. "Technology as experience." interactions 11.5 (2004): 42-43.

2. Garrett, Jesse James. Elements of user experience, the: user-centered design for the web and beyond. Pearson Education, 2010.

3. International Organization for Standardization (2010). Ergonomics of human system interaction - Part 210: Human-centered design for interactive systems. ISO 9241-210:2010

4. International Organization for Standardization (2010). Ergonomics of human-system interaction -- Part 11: Usability: Definitions and concepts. ISO 9241-11

5. Ok, Kerem, et al. "Current benefits and future directions of NFC services." Education and Management Technology (ICEMT), 2010 International Conference on. IEEE, 2010.

6. Massoth, Michael, and Thomas Bingel. "Performance of different mobile payment service concepts compared with a NFC-based solution." Internet and Web Applications and Services, 2009. ICIW'09. Fourth International Conference on. IEEE, 2009.

7. Madlmayr, Gerald, et al. "NFC devices: Security and privacy." 2008 Third International Conference on Availability, Reliability and Security. IEEE, 2008.

8. Tan, Garry Wei-Han, et al. "NFC mobile credit card: the next frontier of mobile payment?." Telematics and Informatics 31.2 (2014): 292-307.

9. Schierz, Paul Gerhardt, Oliver Schilke, and Bernd W. Wirtz. "Understanding consumer acceptance of mobile payment services: An empirical analysis." Electronic commerce research and applications 9.3 (2010): 209-216.

10. Kumar, Poornigha Santhana, Michael Bechinie, and Manfred Tscheligi. "NFC PaymentsGaps Between User Perception and Reality." IFIP International Summer School on Privacy and Identity Management. Springer, Cham, 2017.

11. Kumar, Poornigha Santhana, Michael Bechinie, and Manfred Tscheligi. "Changed the Cup, Not the Saucer-NFC Payments in Supermarkets." International Conference on HumanComputer Interaction. Springer, Cham, 2018

12. Nielsen, Jakob. "Usability inspection methods." Conference companion on Human factors in computing systems. ACM, 1994.

13. Norman, Donald A. The psychology of everyday things. Vol. 5. New York: Basic books, 1988.

14. Ou, Li-Chen, et al. "A study of colour emotion and colour preference. Part I: Colour emotions for single colours." Color Research \& Application 29.3 (2004): 232-240.

15. Lichtlé, Marie-Christine. "The effect of an advertisement's colour on emotions evoked by attitude towards the ad: The moderating role of the optimal stimulation level." International Journal of Advertising 26.1 (2007): 37-62.

16. Hill, Russell A., and Robert A. Barton. "Psychology: red enhances human performance in contests." Nature 435.7040 (2005): 293.

17. Maeda, John. The laws of simplicity. MIT press, 2006.

18. Kitchin, Rob. "'Out of Place','Knowing One's Place': Space, power and the exclusion of disabled people." Disability \& Society 13.3 (1998): 343-356.

19. Bosley, John J. "Creating a short usability metric for user experience (UMUX) scale." Interacting with Computers 25.4 (2013): 317-319. 